\title{
A eficácia da escala de braden na úlcera por pressão em pacientes adultos hospitalizados
}

\author{
Jair Alves Maia ${ }^{1}$ Nilson de Souza dos Santos ${ }^{2} \bullet$ Raquel de Lima Silva $^{3}$
}

As úlceras por pressão são lesões decorrentes de hipóxia celular, levando a necrose tecidual. Geralmente, são localizadas em áreas de proeminências ósseas e ocorrem quando a pressão aplicada à pele, este evento patológico se caracteriza como problema de saúde pública. A construção da Escala de Braden por Braden e Bregstrom foi baseada na fisiopatogenia das úlceras por pressão, através de dois determinantes considerados críticos: a intensidade e a duração da pressão, e a tolerância da pele e das estruturas de suporte para cada força. A intensidade a duração da pressão que o paciente sofre estão relacionadas a mobilidade, atividade percepção sensorial. Por outro lado, a tolerância da pele e das estruturas de suporte estão relacionadas a fatores intrínsecos como nutrição e idade, e a fatores extrínsecos como umidade, fricção e cisalhamento. Com a intenção de colaborar na prevenção de úlceras por pressão, dando subsídios para que os enfermeiros pudessem mais objetivamente indicar quais os pacientes que correm risco para desenvolvê-las, pesquisadores elaboraram escalas, para predizer o risco para sua formação. Este estudo teve como objetivo de identificar a importância da implementação da Escala de Braden na prevenção de úlcera por pressão em pacientes adultos hospitalizados. A metodologia utilizada foi uma revisão clássica da literatura. Portanto, conclui-se que a Escala de Braden consiste em identificar, minimizar, avaliar e reavaliar a pele e sua integridade constantemente pelo enfermeiro. Sua eficiência ratifica a relevância, a confiabilidade e a importância da sua utilização, pois é um poderoso instrumento de avaliação preventiva na úlcera por pressão devendo ser usada pelo enfermeiro.

Palavras-chave: Úlcera por pressão; Escala de Braden; Enfermagem.

${ }^{1}$ Mestrando em Enfermagem pela Faculdade Israelita de Ciências da Saúde Albert Einstein - FICSAE São Paulo (2016), Pós-Graduado em Saúde Pública pela Faculdade Barão do Rio Branco (FAB) (2008), Pós-Graduado em Didática e Docência do Ensino Superior pela Faculdade Barão do Rio Branco (FAB) (2015), graduado em Enfermagem pelo Instituto de Ensino Superior de Manaus (2005).

2 Graduado em Enfermagem pela Faculdade FAMETA (2016).

${ }^{3}$ Enfermeira Graduada em Enfermagem e professora do curso de enfermagem da Faculdade FAMETA (2016). 


\title{
Reação adversa à penicilina cristalina: Síndrome de Lyell
}

\author{
Maria do Carmo Duarte da Costa ${ }^{1} \bullet$ Christielle da Silva Montenegro² $^{2}$
}

Introdução: A necrólise epidérmica tóxica (NET), também denominada síndrome de Lyell, consiste em uma rara patologia dermatológica, na qual a camada superficial da pele se desprende em lâminas, podendo levar à morte.Além da esfoliação mucocutânea, esta doença também se caracteriza pela presença de febre alta e sinais de toxicidade sistêmica. É desencadeada pelo uso de certos fármacos, como penicilina, sulfamidas, barbitúricos, anticonvulsivantes, antiinflamatórios não esteróides ou alopurinol. Da mesma forma que ocorre nos casos de queimaduras graves, a NET pode levar à morte, pois pode haver grande perda de líquido e sais por meio das áreas danificadas, além de deixar o paciente mais susceptível às infecções. Objetivo: Demonstrar evolução clínica de um paciente acometido por NET, no uso prolongado de penicilina cristalina 5.000.000, com diagnóstico diferencial e recuperação favorável. Metodologia: Consiste em um estudo de caso de paciente hospitalizado em uma unidade hospitalar em isolamento de contato após sofrer NET. Resultados: 0 mesmo encontrava-se séptico, desnutrido, anêmico, catabolico, referindo insônia e apresentando depressão. $O$ tratamento tópico foi iniciado com utilização de hidrogel, espuma de poliuretano de prata iônica e espuma de ibuprofeno, com objetivo de realizar desbridamento autolítico, absorção de exsudato e controle de dor. Após oito semanas utilizou-se pomada fitoscar com objetivando-se a cicatrização. A conduta nutricional consistiu em dieta hiperproteica e hipercalórica e uso de imunomoduladores, atingindo-se assim o VET ideal do paciente, de forma que a resposta imunológica fosse melhorada e se estabelecesse um estado anabólico. Conclusão: Ficou evidenciada a necessidade de conhecimento teórico dos profissionais da unidade de internação sobre a NET e o sentido de valor que esta prática pode dar aos profissionais na condução da assistência individualizada aos pacientes sobre seus cuidados, neste estudo, especificamente, os portadores de NET.

Palavras-chave: Hipersensibilidade a Drogas; Manifestações Cutâneas; Dermatopatias.

${ }^{1}$ Nutricionista, Especialista em Nutrição Clínica e Ambulatorial. nutricarmo@hotmai.com

${ }^{2}$ Enf. Especialista em UTI neo e Pediátrica; Graduanda do curso de Esp. Gestão do trabalho e Educação na Saúde e Graduanda o curso de Esp. Enfermagem em Home Care. christellemontenegro@yahoo.com.br 


\title{
A atuação da fisioterapia juntamente com a equipe multiprofissional na cicatrização de lesão por atropelamento, um estudo de caso
}

\author{
Itáira Pereira Menezes Aquino ${ }^{1} \bullet$ Maria Marluce da Silva Oliveira² • Christielle da Silva Montenegro ${ }^{3} \bullet$ \\ Maria do Carmo Duarte da Costa ${ }^{4}$
}

\begin{abstract}
Introdução: 0 trauma por acidente de trabalho é um acontecimento súbito que ocorre no exercício da atividade laboral ao serviço da empresa e que provoque ao trabalhador lesão ou danos corporais de que resulte na incapacidade parcial ou total temporária ou permanente para o trabalho. Objetivo: Apresentar os resultados de uma equipe multiprofissional na cicatrização e reabilitação física funcional de uma lesão por atropelamento. Metodologia: Consiste em um estudo de caso de uma paciente acompanhada por equipe multiprofissional, que sofreu um trauma por uma empilhadeira no quadríceps da perna direita. Resultados: Paciente admitida no dia 02/11/15, vítima de acidente de trabalho onde foi acometida por uma empilhadeira agredindo quadríceps direito com perca de $90 \%$ da massa muscular. Segundo avaliação da enfermagem, a ferida apresentava $80 \%$ de tecido necrótico e $20 \%$ de tecido de granulação, com nível alto de exsudação, aspecto seroso, presença de odor detectado na remoção da cobertura. Tratamento foi iniciado com antisséptico com clorexine $2 \%$, soro fisiológico morno, cobertura de carvão ativado e hidrogel. Posteriormente foi usado alginato de prata e Biatain Ibu. No âmbito nutricional foi realizada avaliação física, onde se constatou que a paciente estava anêmica, com alto catabolismo e inapetente. A conduta consistiu em oferta de fórmula anti-anêmica composta de quelatos, suplemento de arginina, glutamina, mix de colágeno composto de micronutrientes, além de dieta hipercalórica e hiperproteica. Foi ressaltada a importância da hidratação adequada e da manutenção dos hábitos alimentares. Após avaliação da fisioterapeuta pode ser observado engurgitamento de linfa, edema grau III, atrofia por desuso, perda da sensibilidade, hiporreflexia, mialgia, rigidez articular, diminuição da amplitude de movimento e circulação comprometida na extremidade do membro afetado. 0 primeiro procedimento realizado foi à massagem miofacial com ativação da linfa e drenagem linfática, seguido pelo desenvolvimento de exercícios para amplitude de movimento como: alongamento e mobilizações. Paciente seguiu evoluindo, desenvolvendo o tratamento através de exercícios passivos, ativo-assistido, ativo, descarga de peso, treino da marcha e resistência. Atualmente, vem sendo realizada um tratamento dermato-funcional que visa à melhora das características da lesão através da fricção. Como resultado do tratamento multiprofissional, incluindo da fisioterapia, paciente encontra-se deambulando sem necessidade de apoio e sem prejuízo nos movimentos, foi recuperada a sensibilidade, a circulação periférica, também houve melhora do tônus muscular, a ferida apresenta $80 \%$ já cicatrizado e $20 \%$ de epitelização. Nutricionalmente, paciente apresenta-se sem nenhum déficit nutricional além do apetite que foi recuperado. Conclusão: Com a ação multiprofissional no tratamento da lesão, observou-se a recuperação total da função sensitiva e motora do membro inferior direito, bem como do tecido celular subcutâneo, epiderme e derme, sendo portanto, desnecessário o implante de pele no local.
\end{abstract}

Palavras-chave: Reabilitação funcional; Coberturas especiais; Arginina; Nutrição imunomoduladora.

${ }^{1}$ Fisioterapeuta, Especialista em Ortopedia, traumatologia e desportiva e Instrutora de Pilates. Avançado. itairamenezes@gmail.com

2 Médico Clínico Geral, Pós-Graduada em doenças da pele.marla_med07@hotmail.com

${ }^{3}$ Enf. Especialista em UTI neo e Pediátrica; Graduanda do curso de Esp. Gestão do trabalho e Educação na Saúde e Graduanda o curso de Esp. Enfermagem em Home Care. christellemontenegro@yahoo.com.br

${ }^{4}$ Nutricionista, Especialista em Nutrição Clínica e Ambulatorial. nutricarmo@hotmail.com 


\title{
Construção do fluxo dos usuários do programa assistencia à pacientes portadores de feridas e sua utilidade no planejamento da emad (equipe multiprofissional de atendimento domiciliar)
}

Deyvidy Albuquerque de Oliveira ${ }^{1} \bullet$ Christielle da Silva Montenegro $^{2} \bullet$ Alcineia Pereira Lira Camurça $^{3} \bullet$ Raisa de Paula Oliveira ${ }^{4}$

\begin{abstract}
Objetivo: Montar o fluxograma por onde os usuários do Programa de Pacientes Portadores de Feridas percorrem para uso no Planejamento da EMAD e das Unidades de Saúde. Método: Construção em conjunto com a Coordenadora da EMAD (Equipe Multiprofissional de Atendimento Domiciliar), Christielle Montenegro, do Fluxo. Resultados: Foram identificadas as portas de entrada do usuário no programa e atores, instituições e profissionais, envolvidos, abrindo caminho para uso no Planejamento da EMAD. Conclusão: A importância de se conhecer os processos envolvidos no percurso por onde o usuário percorre influencia em tomadas de decisões de todos os atores envolvidos no serviço prestado. Conhecer o Fluxo por onde o usuário portador de feridas percorre no serviço é uma ferramenta para a utilização do PES (Planejamento Estratégico Situacional) bem como conhecer os vértices do Triângulo de Governo em cada etapa por onde o usuário percorre e trabalhar em cima de resultados que se pretende alcançar, prever problemas que possam vim a surgir e resolver problemas já existentes.
\end{abstract}




\title{
Liga acadêmica de assistência a pacientes portadores de lesões
}

\author{
Yara de Moura Magalhães ${ }^{1} \bullet$ Suellen Cristina Enes Valentim da Silva ${ }^{2}$ Thaisa Castello Branco Danzicourt ${ }^{3} \bullet$ \\ Eline Messias ${ }^{4} \bullet$ Barbara Teles Cameli $^{5}$
}

Introdução: Diferentemente de boa parte das alterações de pele, as lesões tem sido alvo de grande preocupação para os serviços de saúde, pois sua ocorrência causa impacto tanto para os pacientes e seus familiares, quanto para o próprio sistema de saúde, com o prolongamento de internações, riscos de infecções e outros agravos evitáveis. O tratamento de lesões é um processo complexo e desafiador, envolvendo uma equipe multiprofissional, como clínicos, nutricionistas, fisioterapeutas, enfermeiras especializadas, entre outros, tornando-se essencial para o adequado tratamento dos pacientes. Objetivo: Oferecer aos portadores de feridas (residentes na cidade de Rio Branco, Acre), ações educativas, preventivas e curativas nas áreas de enfermagem e nutrição. Metodologia: As atividades desenvolvidas ocorrerão através do vinculo da Equipe Multiprofissional de Atenção Domiciliar com a Universidade Federal do Acre que realizará uma seleção de acadêmicos para compor a equipe da Liga Acadêmica, juntamente com os docentes. Resultados: Como liga acadêmica, serão desenvolvidas atividades de cunho prático e teórico. As atividades teóricas consistem na realização de reunião para estudo de casos, discussão de artigos, planejamento de atividades, exposição de aulas sobre os temas e outros, já as atividades práticas serão realizadas com o acompanhamento dos acadêmicos a equipe do EMAD. Nesse primeiro momento, serão selecionados acadêmicos do curso de nutrição, que iniciarão a vivência, aprendendo na prática sobre lesões, terapia nutricional, cuidados domiciliares, etc. Todas as atividades seguindo o protocolo de atendimento já existente no EMAD, que consiste na primeira avaliação, seguida pela realização do curativo em domicílio e a oferta da terapia nutricional na atenção domiciliar. Os acadêmicos irão acrescentar a equipe com cuidados teóricos como a orientação familiar quanto à higiene, entrega de plano alimentar individualizado, cálculo da estimativa de necessidade energética, entre outros. Estando estabelecida essa primeira fase, a liga se expandirá para os demais cursos, como enfermagem, psicologia, medicina, entre outros. Conclusão: Almeja-se promover através do ensino, pesquisa e extensão a aproximação da academia ao ambiente familiar desses pacientes, atingindo também a comunidade. Intervindo com ações de conscientização, prevenção e recuperação da saúde, contribuindo para a qualidade de vida e capacidade funcional do paciente. Com as visitas domiciliares, esperamos realizar um levantamento de dados epidemiológicos para que possamos em longo prazo estabelecer um perfil da incidência e prevalência de pacientes acometidos com lesões na cidade de Rio Branco.

Palavras-chave: Liga Acadêmica; Portadores de Feridas; Equipe Multiprofissional.

${ }^{1}$ Universidade Federal do Acre. ymagalhaes9@gmail.com

${ }_{2}^{2}$ Universidade Federal do Acre.suellencris_czs@hotmail.com

3 Universidade Federal do Acre. thaisadanz@gmail.com

${ }^{4}$ Prof. Esp. Centro de Ciências da Saúde e do Desporto da Universidade Federal do Acre. eline_nutri@yahoo.com.br

${ }^{5}$ Prof. Esp. Centro de Ciências da Saúde e do Desporto da Universidade Federal do Acre.babi_cameli@hotmail.com 